\title{
New Surgical Options in Glaucoma
}

Lisa V. Heckler, MD, FRCSC, Département d'ophtalmologie, Université de Montréal, Montreal, Quebec.

Michael W. Dorey, MD, FRCSC, Department of Ophthalmology and Visual Sciences, University of Alberta, Edmonton, Alberta.

Karim F. Damji, MD, FRCSC, MBA, Department of Ophthalmology and Visual Sciences, University of Alberta, Edmonton, Alberta.

Correspondence may directed to: kdamji@ualberta.ca

2319, 10240 Kingsway Avenue NW, Edmonton, AB T5H 3V9 Canada

\begin{abstract}
The treatment of glaucoma is undergoing constant change. In the last decade, there has been a surge of novel surgical options that aim to lower intraocular pressure while providing improved safety profiles compared to traditional incisional glaucoma surgery. This article summarizes four such options - trabectome, iStent, canaloplasty and endocyclophotocoagulation — including descriptions of the procedures and evidence behind them.
\end{abstract}

Key Words: Micro-invasive glaucoma surgery, ab interno trabeculectomy, trabecular micro-bypass stent, canaloplasty, endocyclophotocoagulation

\section{Résumé}

Le traitement du glaucome est en constante évolution. Au cours de la dernière décennie, on a observé un afflux de nouvelles options chirurgicales qui visent à abaisser la pression intraoculaire tout en présentant une innocuité accrue par rapport à la chirurgie classique par incision. Cet article décrit quatre de ces options - atrabéculectomie endoculaire au Trabectome ${ }^{\circledR}$, mise en place du micro-implant iStent ${ }^{\circledR}$, canaloplastie et cyclophotocoagulation endoscopique -, y compris leur déroulement et les données qui les étayent.

Key Words: Chirurgie micro-invasive du glaucome, trabéculectomie endoculaire, micro-implant de dérivation trabéculaire, canaloplastie, cyclophotocoagulation endoscopique

\section{Introduction}

Glaucoma is the second leading cause of blindness globally after cataract. ${ }^{1}$ Treatment of glaucoma aims to preserve visual function and maintain overall quality of life. ${ }^{2}$ In Canada, a model of interprofessional collaboration between ophthalmologists and optometrists has been suggested. ${ }^{3}$ Management decisions are made based on the mechanism of glaucoma, the stage of disease, and the degree of intraocular pressure (IOP) elevation. Patients and care partners should be approached in an individualized manner, taking into account their biopsychosociospiritual (BPSS) profile and preferences. ${ }^{4}$ This approach considers various components of health, including systemic biology such as life expectancy, psychological factors, socioeconomic considerations, and spiritual/cultural values.
The standard treatment algorithm has typically employed medications, laser treatment, and eventually surgery. Medications available in Canada include single agents and combination agents involving prostaglandin analogues, beta-blockers, alpha-agonists, carbonic anhydrase inhibitors, and miotics. Laser treatment options include argon laser trabeculoplasty, selective laser trabeculoplasty, and in more severe cases cyclophotocoagulation. This paper discusses surgical options for glaucoma, in particular several newer and less invasive techniques. We will be focusing on the trabectome, iStent, and endocyclophotocogulation, which are $a b$ interno techniques, as well as canaloplasty, an ab externo technique. There are already several studies reviewing these techniques and any evidence supporting them, and we have referenced these works throughout this paper. ${ }^{5-7}$ 

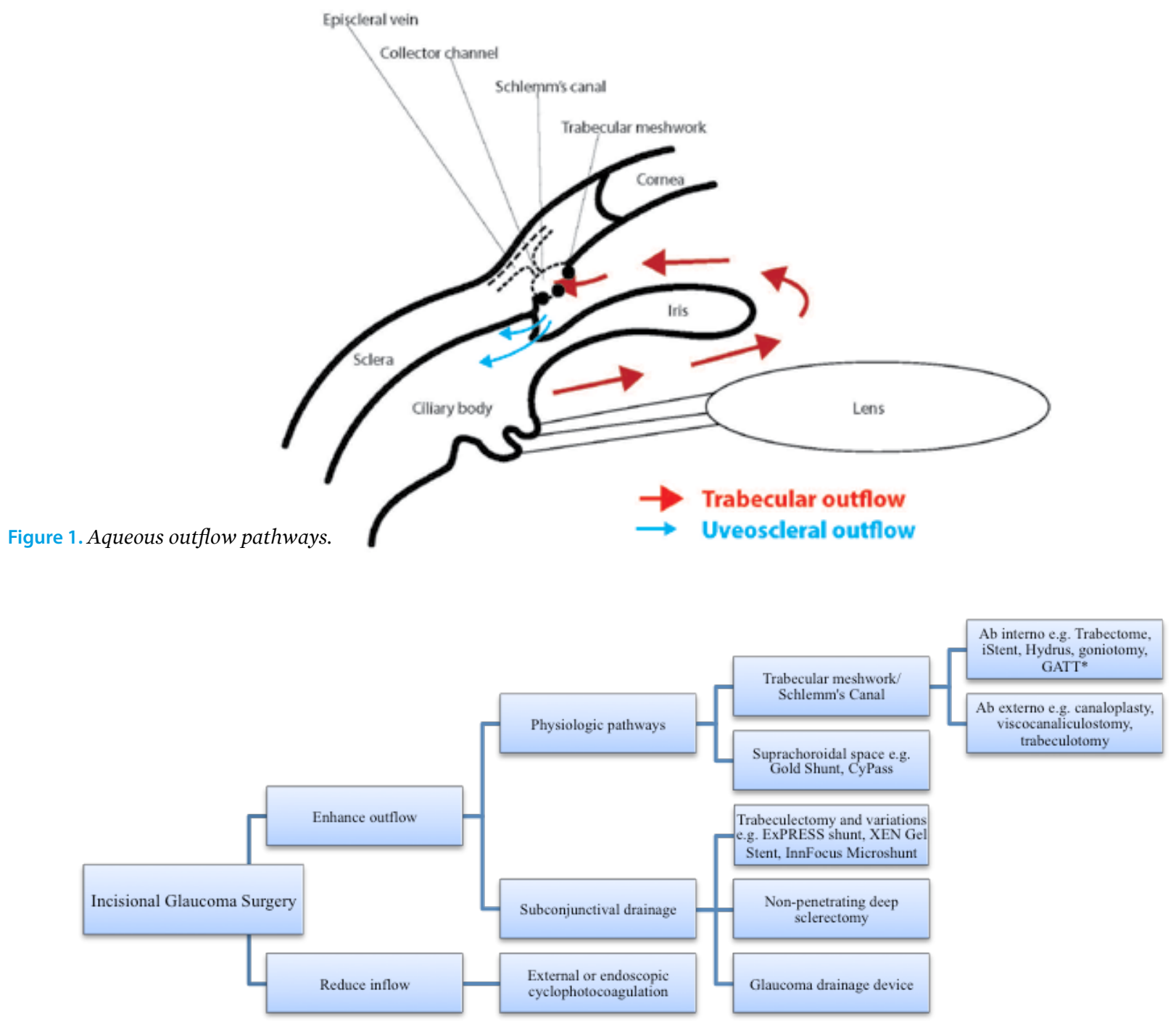

Figure 2. Classification of Incisional Glaucoma Surgery. * Gonioscopy-assisted transluminal trabeculotomy

\section{Glaucoma Surgery: A Brief History}

Since the 1950s, we have known that the major site of aqueous outflow resistance is the juxtacanalicular portion of the trabecular meshwork. ${ }^{8}$ Efforts were made as early as the 1950 s to perforate this tissue with energy from light sources to allow for improved outflow. ${ }^{9}$ Moses studied microsurgical electro cautery techniques to the trabecular meshwork in enucleated eyes in $1971 .{ }^{10}$ Shortly thereafter, laser treatment of the trabecular meshwork became well studied and employed. Surgery to the angle has been well established in the congenital glaucoma population, but it was not until the last fifteen years that angle surgery in adults has come into favour. ${ }^{11}$

\section{A Review Of The Relevant Anatomy}

The aqueous is produced by the ciliary processes and leaves the eye by two main pathways: the conventional or trabecular pathway, accounting for $70-95 \%$ of aqueous egress from the eye, and the unconventional or uveoscleral outflow, accounting for the other 5-30\% (see Figure 1). ${ }^{12}$ The techniques described here will be focusing on the aqueous production and trabecular outflow. This consists of the trabecular meshwork, Schlemm's canal, intrascleral channels, and the episcleral or conjunctival veins.

\section{Classification Of Incisional Surgeries For Glaucoma}

Incisional glaucoma surgery can be divided into procedures that reduce inflow of aqueous and procedures that enhance outflow (see Figure 2). Outflow procedures are the most common. Currently, trabeculectomy and glaucoma drainage devices remain the most frequently used techniques and several large-scale studies have demonstrated their efficacy. ${ }^{13-15}$ Over the last ten years, there has been a shift towards 
alternative glaucoma surgeries, largely due to the high rate of complications associated with traditional glaucoma surgeries. These newer techniques have become known as "microinvasive glaucoma surgery," or MIGS, and play a different role in the glaucoma treatment algorithm. ${ }^{5,7,11}$

\section{Enhancing the Outflow Pathways: Trabectome, iStent, and Canaloplasty Trabectome}

\section{Description of the Procedure}

The Trabectome (Neomedix Corp.) is composed of a disposable hand piece that supplies irrigation and aspiration and a tip that delivers high-frequency electro cautery. The hand piece is advanced across the anterior chamber through a temporal corneal incision towards the nasal angle with the irrigating fluid on. Under gonioscopic visualization, the footplate is inserted into the trabecular meshwork. The cautery is activated, and the surgeon advances the instrument in a clockwise and counterclockwise manner, thereby removing a 3-4 clock-hour arc of trabecular meshwork and the inner wall of Schlemm's canal. This effectively creates a direct pathway for the aqueous to flow from the anterior chamber to the collector channels. ${ }^{6}$ The procedure is generally combined with cataract extraction, although there may be certain subgroups who do well with trabectome alone, such as exfoliation syndrome, ${ }^{16}$ and juvenile open angle glaucoma. ${ }^{17}$

The advantages of this procedure over traditional glaucoma surgeries include the faster recovery, due to the less invasive nature, the approach which leaves the conjunctiva intact for future surgeries, the lack of a filtering bleb, and the minimal added risk to the patient if combined with phacoemulsification. ${ }^{18}$ The disadvantages are that IOP lowering is generally not as substantial as it is with traditional glaucoma surgery, and there are some potential complications, the most common one being hyphema. This usually clears in a few days. Other complications include damage to the cornea, iris, or lens, as well as intraocular pressure elevation, but serious complications such as hypotony and suphrachoroidal hemorrhage are very rare. ${ }^{19}$ Delayed hyphema has also been reported ${ }^{20}$ where blood refluxes through the trabectome cleft, which usually resolves within a few weeks but can recur.

\section{Evidence}

Several studies have evaluated the effectiveness of trabectome, the first ones being trabectome alone ${ }^{21}$ and later ones being trabectome combined with phacoemulsification. ${ }^{22}$ The first large study was a noncomparative prospective case series of 304 eyes with open-angle glaucoma undergoing trabectome combined with cataract extraction..$^{22}$ Mean baseline IOP of 20
$\mathrm{mmHg}$ decreased at 12 months to $15.5 \mathrm{mmHg}$, and medications decreased from 2.65 to 1.76 . Secondary glaucoma procedures were performed in 9 patients. A retrospective review of 88 cases of trabectome alone and 158 cases of combined trabectome and cataract from the Mayo Clinic, found a reduction in mean IOP from $21.6 \mathrm{mmHg}$ to $15.3 \mathrm{mmHg}$ and a decrease in number of glaucoma medications from 3.1 to 1.9 at 2 years. However, subsequent glaucoma surgery was required in 66 patients $(26.8 \%)$, an average of 10 months after surgery. ${ }^{23}$ The weakness of these studies is the lack of a comparison (e.g. phacoemulsification alone) to prove the effectiveness of the procedure, since prior studies have demonstrated the IOPlowering benefit of phacoemulsification alone..$^{24,25}$

\section{iStent}

\section{Description of the Procedure}

The Glaukos micro-bypass trabecular iStent (Glaukos Corp.) is the smallest medical device to be implanted in the human body. It is made of nonferromagnetic heparin coated titanium and consists of an inlet ("snorkel") connected at a right angle to the implantable portion (see Figure 3). ${ }^{6}$ The preloaded iStent inserter is advanced across the anterior chamber through a temporal corneal incision under gonioscopic visualization towards the nasal angle. The sharp tip of the iStent is used to engage and perforate the trabecular meshwork, and the stent then slides into the Schlemm canal. The device is then released from the inserter. Usage of the iStent has been studied in combination with cataract extractions. The advantages of the iStent are similar to the trabectome in terms of minimizing ocular tissue damage, and possibly lower risk of complications. ${ }^{26}$

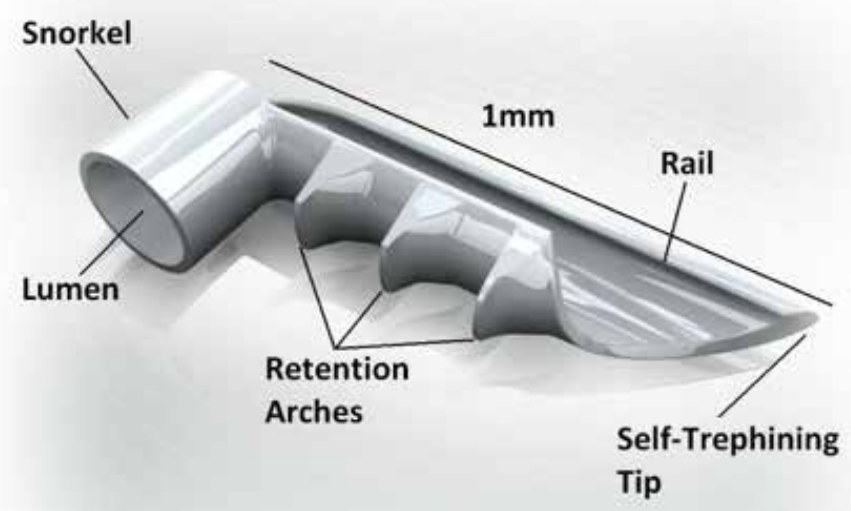

Figure 3. : iStent Trabecular Microbypass Stent. Courtesy of Glaukos Corporation, Laguna Hills, California, USA. 


\section{Evidence}

Initial studies reported the use of a single iStent per eye, though subsequent studies found multiple stents to be more effective. ${ }^{27}$ Spiegal et al. reported the successful use of the iStent in 2008 in a prospective, multicenter noncomparative interventional trial..$^{28}$ Since then, further validation has arisen from randomized control trials comparing phacoemulsification alone to phacoemulsification with iStents. ${ }^{29-31}$ In the two-year follow-up paper by the iStent Study Group, 240 eyes with mild to moderate glaucoma were randomized to receive one iStent combined with phacoemulsification or phacoemulsification alone. At both one and two years, the proportion of patients with unmedicated IOP of 21 or less was significantly higher in the iStent group. ${ }^{31}$ Amount of hypotensive medications were lower in the stent group at one year, but this did not reach statistical significance at two years. Complication rate was similar between the two groups, and there were no stent-related adverse effects reported after two years. More recently, Ahmed et al. evaluated the use of two iStents combined with travaprost post-operatively, to improve both conventional trabecular and uveoscleral outflow in 39 open angle glaucoma patients uncontrolled on two medications. Mean IOP decreased from $22.2 \mathrm{mmHg}$ to $11.8 \mathrm{mmHg}$ at 18 months, from two medications pre-operatively to one medication post-operatively. ${ }^{32}$

Further expansion of the iStent's role and its long-term efficacy are yet to be determined. Some authors have suggested it may play a limited role in select patients with advanced disease or even prior filtering surgery. ${ }^{33,34}$

\section{Canaloplasty}

\section{Description of the Procedure}

This technique is similar in concept to viscocanalostomy, which involves the creation of a deep sclerectomy followed by injection of viscoelastic into Schlemm's canal. In canaloplasty, a superficial and then deeper scleral flap is created, so that Schlemm's canal can be reached. The canaloplasty device (Ellex) contains a fiber optic light catheter that is then inserted into one end of the open Schlemm's canal. This is threaded around the canal for 360 degrees, with the aid of the light, such that a false passage is not created, until it exits from the other opening in Schlemm's canal. A prolene suture is then attached to the distal end of the catheter, and the catheter is withdrawn, thereby threading the suture through the canal. The ends of the suture are tied to distend the trabecular meshwork.

The advantages of this procedure are that it does not require a filtering bleb, though occasionally one forms, ${ }^{35}$ and it has less complications compared to conventional trabeculectomy. The disadvantages are that the learning curve is long, in some cases it is impossible to canulate Schlemm's canal, and the IOP-lowering effect is not as dramatic as trabeculectomy. ${ }^{36}$ It is also more invasive than the MIGS procedures, as it disrupts conjunctiva, although $a b$ interno approaches are now also being explored.

\section{Evidence}

In a nonrandomized multicentre trial, Lewis et al. reported the three-year results of 157 patients undergoing canaloplasty or combined canaloplasty-cataract surgery. ${ }^{35}$ Baseline IOP of 28.5 $\mathrm{mmHg}$ reduced to $15.2 \mathrm{mmHg}$, and medications decreased from 1.8 to 0.8 . Most complications occurred intraoperatively or in the early post-operative period, including partial suture extrusion through the trabecular meshwork, Descemet's detachment, hyphema, and IOP elevation.

Brüggemann et al. also compared canaloplasty to trabeculectomy. ${ }^{37}$ In this study of 15 patients with prior trabeculectomy in one eye, the contralateral eye underwent canalopasty, and 6- and 12-month results were compared. Both procedures reduced the IOP significantly; however, the trabeculectomy group achieved lower IOP (11.64 vs. $13.21 \mathrm{mmHg}$ ), was on no medication, and required fewer additional interventions. However, they required longer initial hospitalization and more post-operative visits.

\section{Reducing Inflow: Endoscopic Cyclophotocoagulation Description of the Procedure}

Cyclophotocoagulation was first used in the 1970s to lower intraocular pressure. It is usually done through a contact approach delivered over the area of the ciliary body, thereby reducing aqueous production. It is generally left as a last-resort to treat end-stage glaucoma due to its unpredictability and

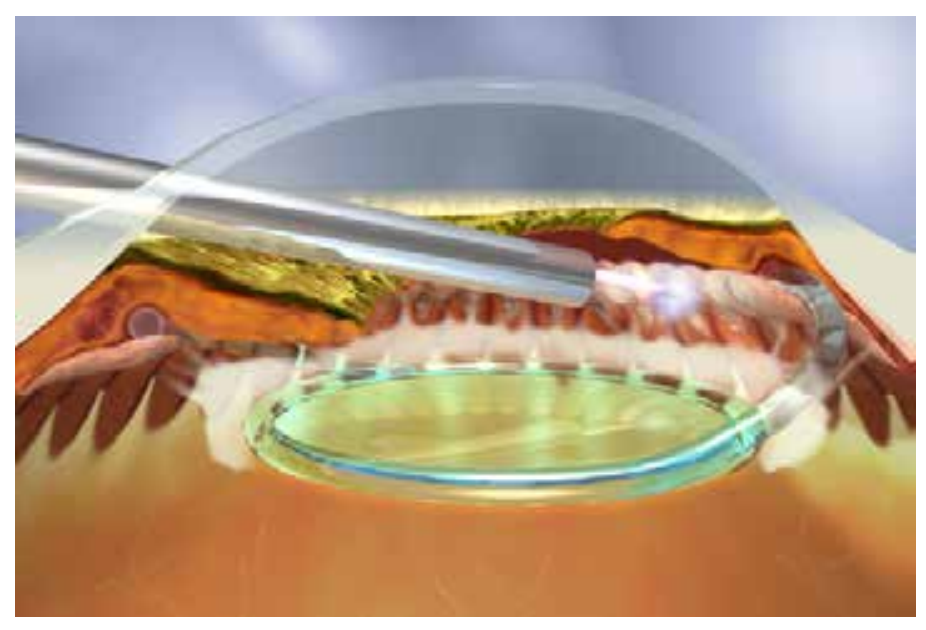

Figure 4.: Endoscopic Cyclophotocoagulation of the Ciliary Processes. Courtesy of Endo Optiks, Inc., Little Silver, New Jersey, USA. 
possibility of complications like phthisis. ${ }^{38}$ In 1992, however, Uram described the use of photocoagulation delivered to the ciliary body under direct visualization with endoscopy. ${ }^{39}$

Endoscopic cyclophotocoagulation (ECP) has been used in several different types of glaucoma, including primary open angle, neovascular glaucoma, chronic angle closure, uveitic, angle recession, ${ }^{38}$ and a less destructive version in plateau iris syndrome. ${ }^{40}$ The laser unit contains a probe to deliver the diode laser that has a built-in endoscope and attached camera monitor. When this is combined with phacoemulsification, the ECP is generally done before or after the lens is inserted. The probe is inserted through a corneal incision and directed behind the iris until the ciliary processes come into view on the camera monitor. The laser is then fired with a foot pedal and the desired outcome is a whitening of the ciliary processes (see Figure 4). This treatment can be done for a circumference of 90-360 degrees, and a second incision may be required..$^{38}$

\section{Evidence}

A retrospective review of 368 eyes that underwent phacoemulsification combined with ECP found a $10.9 \mathrm{mmHg}$ drop in IOP at two years post-operatively, with a mean decrease in number of medications of $1.1 .^{41}$ The largest published study, by the same group, was a retrospective review of 539 patients. ${ }^{42}$ All eyes had at least one prior glaucoma surgery and IOP $\geq$ 35 on maximal medical therapy or advanced glaucoma with IOP above target. The mean IOP decreased from a baseline of $38 \mathrm{mmHg}$ to $12.1 \mathrm{mmHg}$, and an average number of medications of 1.9 , after 5 years. Prospective studies have also evaluated the use of ECP. In a randomized trial, Gayton et al. compared ECP to trabeculectomy and found a $29 \%$ reduction in IOP after ECP vs. 32\% following trabeculectomy, with similar baseline IOP of $25 \mathrm{mmHg} .{ }^{43}$ Francis et al. evaluated ECP use after failed aqueous shunts. Twent-five eyes received ECP for 360 degrees. ${ }^{44}$ Success, defined as reduction in IOP of $3 \mathrm{mmHg}$ and discontinuation of nontolerated glaucoma medications, was achieved in $88 \%$ of patients up to two years follow up. Murthy et al. studied 50 eyes of Indian patients with refractory glaucoma, including phakic, pseudophakicm and phakic patients, in a prospective non-comparative study. ${ }^{45} \mathrm{IOP}$ decreased from $32.58 \mathrm{mmHg}$ to $13.96 \mathrm{mmHg}$, with an average follow up of about one year. The average number of glaucoma medications decreased from 2.51 to 1.09 .

\section{How To Choose Which Procedure To Use On On Each Patient?}

The decision to proceed with glaucoma surgery is a complex one that should take into account different ocular and systemic factors. In the Canadian model, where optometrists and ophthalmologists work together in the best interest of patients, effective and timely communication is essential. ${ }^{3,46}$ Treatment of glaucoma and glaucoma suspects is well summarized in the Canadian Ophthalmological Society evidence-based clinical practice guidelines for the management of glaucoma in the adult eye. ${ }^{2}$ Indications for surgery include situations where other methods of lowering IOP have been unsuccessful and the optic nerve is failing or likely to fail. Furthermore, surgery should be considered when patients are intolerant to and/or non-compliant with medications. Referral to an ophthalmologist when progression is suspected is crucial in preventing glaucoma-related blindness.

The indications for some of the novel glaucoma surgeries described here are currently in evolution. The procedures that act by increasing outflow (trabectome, iStent, canaloplasty) have primarily been studied in the context of mild to moderate glaucoma and are usually combined with cataract surgery. Since they generally do not lower IOP to a level as low as traditional surgery, the authors favour their use in situations where there is a visually significant cataract and early or moderate glaucoma where the IOP is above target. ECP, however, may be a good option in more advanced or refractory glaucoma based on the available literature.

Other factors that might encourage a surgeon to proceed with a MIGS over a trabeculectomy or tube shunt include poor conjunctival or scleral tissue, where a trabectome or iStents would be safer than an external surgery, as well as risk factors for over-filtration (e.g. myopia) or suprachoroidal hemorrhage (e.g. older age, presence of hypertension, use of anticoagulants). Fortunately, most MIGS procedures do not seem to affect the future outcome of traditional glaucoma surgery. ${ }^{47}$ Finally, patients and care partners should be involved in the discussion and decision-making regarding treatment.

\section{Post-Operative Care}

Post-operative care for microinvasive glaucoma surgeries is similar to post-operative cataract care; however, there are several added complications that should be watched for. Uveitis may be more prominent due to the added intraocular manipulation, in particular with ECP. ${ }^{38}$ This can usually be managed with steroid drops and/or non-steroidal agents, especially to reduce the risk of cystoid macular edema. Hyphema or microhyphema is not uncommon and can be treated conservatively with steroid drops, reduced level of activity, and elevation of the head. If a hyphema is large (i.e. over one-half of the anterior chamber), not clearing after two weeks, or associated with elevated intraocular pressure, 
consideration should be given to a surgical washout.

Intraocular pressure elevation may occur, with early pressure rise likely due to inflammation and later rise more likely from steroid usage, particularly after trabectome (author's personal experience) or iStent surgery. ${ }^{48}$ The management of these two situations, therefore, differs. If the inflammatory response seems prominent, steroids should be increased; however, if steroid-induced pressure elevation is suspected and inflammation is minimal, lower-potency steroids or non-steroidal agents should be used and rapidly tapered off.

Since some of these procedures include the insertion of a device, there is also the potential for such device-related complications as obstruction or malposition, though these risks are low. ${ }^{5}$ Gonioscopy may be necessary to identify these issues. Finally, as is the case with all glaucoma surgeries, the pressure control may decrease or fail over time, at which point other methods must be employed (drops, laser, further surgery).

\section{Conclusion And Summary}

Surgical care of glaucoma is undergoing some major paradigm shifts. Larger scale randomized control trials will be important in better defining the role of MIGS in the treatment algorithms. Other MIGS procedures not discussed here have also been described, including Hydrus, CyPass, iStent inject (secondgeneration iStent), as well as other newer surgical treatments such as the Gold shunt. Finally, modifications of conventional surgeries have been studied and employed by many, including the Ex-PRESS Glaucoma Filtration Device (Alcon, Inc.), as an additive device to traditional trabeculectomy surgery. With continued collaboration among eye care specialists, individualized glaucoma care is becoming a reality.

\section{References}

1. Resnikoff S, Pascolini D, Etya'ale D, et al. Global data on visual impairment in the year 2002. Bull World Health Organ 2004;82:844-51.

2. Canadian Ophthalmological Society Glaucoma Clinical Practice Guideline Expert Committee, Canadian Ophthalmological Society. Canadian Ophthalmological Society evidence-based clinical practice guidelines for the management of glaucoma in the adult eye. Can J Ophthalmol 2009;44(Suppl 1):S7-S93.

3. Canadian Glaucoma Society Committee on Interprofessional Collaboration in Glaucoma Care. Model of interprofessional collaboration in the care of glaucoma patients and glaucoma suspects. Can J Ophthalmol 201;46(6 Suppl):S1-S21.

4. Gessesse GW, Damji KF. Advanced glaucoma: management pearls. Middle East Afr J Ophthalmol 2013;20:131-41.

5. Saheb H, Ahmed II. Micro-invasive glaucoma surgery: current perspectives and future directions. Curr Opin Ophthalmol 2012;23:96-104.

6. Francis BA, Singh K, Lin SC, et al. Novel glaucoma procedures: a report by the American Academy of Ophthalmology. Ophthalmology 2011;118:1466-80.

7. SooHoo JR, Seibold LK, Radcliffe NM, et al. Minimally invasive glaucoma surgery: current implants and future innovations. Can J Ophthalmol 2014;49:528-33.
8. Grant WM. Facilities of flow through the trabecular meshwork. Arch Ophthalmol 1955;54:245-8.

9. Van Buskirk EM, Shields MB. 100 Years of Progress in Glaucoma. Philadelphia: Lippincott-Raven; 1997.

10. Moses RA. Electrocautery puncture of the trabecular meshwork in enucleated human eyes. Am J Ophthalmol 1971;72:1094-6.

11. Kahook MY, Salim S, Seibold LK. MIGS: Advances in Glaucoma Surgery. Thorofare: SLACK Inc.; 2014.

12. Allingham RR, Damji KF, Freedman S, et al. Shields Textbook of Glaucoma. 6th ed. Philadelphia: Lippincott Williams \& Wilkins; 2011.

13. AGIS Investigators. The Advanced Glaucoma Intervention Study (AGIS): 9. Comparison of glaucoma outcomes in black and white patients within treatment groups. Am J Ophthalmol 2001;132:311-20.

14. Musch DC, Gillespie BW, Niziol LM, et al. CIGTS Study Group. Intraocular pressure control and long-term visual field loss in the Collaborative Initial Glaucoma Treatment Study. Ophthalmology 2011;118:1766-73.

15. Gedde SJ, Schiffman JC, Feuer WJ, et al. Three-year follow-up of the tube versus trabeculectomy study. Am J Ophthalmol 2009;148:670-84.

16. Ting JL, Damji KF, Stiles MC, et al. Ab interno trabeculectomy: outcomes in exfoliation versus primary open-angle glaucoma. J Cataract Refract Surg 2012;38:315-23.

17. Damji KF, Arora S, Masahiro M, et al. Efficacy and Safety of Ab Interno Trabeculectomy with Trabectome in Juvenile Open Angle Glaucoma (JOAG). Presented at: American Glaucoma Society (AGS) Annual Meeting; 2013 Feb 28-Mar 3; San Francisco, CA.

18. Filippopoulos T, Rhee DJ. Novel surgical procedures in glaucoma: advances in penetrating glaucoma surgery. Curr Opin Ophthalmol 2008;19:149-54.

19. Minckler D, Mosaed S, Dustin L, et al. Trabectome (trabeculectomy-internal approach): additional experience and extended follow-up. Trans Am Ophthalmol Soc 2008;106:149-59; discussion; pp. 159-60.

20. Ahuja Y, Malihi M, Sit AJ. Delayed-onset symptomatic hyphema after ab interno trabeculotomy surgery. Am J Ophthalmol 2012;154:476-80.e2.

21. Minckler D, Baerveldt G, Ramirez MA, , et al. Clinical results with the Trabectome, a novel surgical device for treatment of open-angle glaucoma. Trans Am Ophthalmol Soc 2006;104:40-50.

22. Francis BA, Minckler D, Dustin L, et al. Combined cataract extraction and trabeculotomy by the internal approach for coexisting cataract and openangle glaucoma: initial results. J Cataract Refract Surg 2008;34:1096-103.

23. Ahuja Y, Ma Khin Pyi S, Malihi M, et al. Clinical results of ab interno trabeculotomy using the trabectome for open-angle glaucoma: the Mayo Clinic series in Rochester, Minnesota. Am J Ophthalmol 2013;156:927-35.e2.

24. Mathalone N, Hyams M, Neiman S, et al. Long-term intraocular pressure control after clear corneal phacoemulsification in glaucoma patients. J Cataract Refract Surg 2005;31:479-83.

25. Damji KF, Konstas AG, Liebmann JM, , et al. Intraocular pressure following phacoemulsification in patients with and without exfoliation syndrome: a 2 year prospective study. Br J Ophthalmol 2006;90:1014-8.

26. Kurji K, Arora S, Rudnisky CJ, et al. Phaco-Trabectome vs. Phaco-iStent in Patients with Open-Angle Glaucoma. Poster session presented at: American Academy of Ophthalmology (AAO) Annual Meeting; 2013 Nov 16-19; New Orleans, LA.

27. Belovay GW, Naqi A, Chan BJ, et al. Using multiple trabecular micro-bypass stents in cataract patients to treat open-angle glaucoma. J Cataract Refract Surg 2012;38:1911-7.

28. Spiegel D, Garcia-Feijoo J, Garcia-Sanchez J, et al. Coexistent primary openangle glaucoma and cataract: preliminary analysis of treatment by cataract surgery and the iStent trabecular micro-bypass stent. Adv Ther 2008;25:453-64.

29. Fea AM. Phacoemulsification versus phacoemulsification with micro-bypass stent implantation in primary open-angle glaucoma: randomized doublemasked clinical trial. J Cataract Refract Surg 2010;36:407-12.

30. Samuelson TW, Katz LJ, Wells JM, US iStent Study Group, et al. Randomized evaluation of the trabecular micro-bypass stent with phacoemulsification in patients with glaucoma and cataract. Ophthalmology 2011;118:459-67. 
31. Craven ER, Katz LJ, Wells JM, iStent Study Group, et al. Cataract surgery with trabecular micro-bypass stent implantation in patients with mild-tomoderate open-angle glaucoma and cataract: two-year follow-up. J Cataract Refract Surg 2012;38:1339-45.

32. Ahmed II, Katz LJ, Chang DF, , et al. Prospective evaluation of microinvasive glaucoma surgery with trabecular microbypass stents and prostaglandin in open-angle glaucoma. J Cataract Refract Surg 2014;40:1295-300.

33. Arora S, Rudnisky C, Kurji K, et al. Phaco-Trabecular Micro-Bypass Stent in Management of Patients with Advanced Open-Angle Glaucoma. Paper presentation at: American Society of Cataract and Refractive Surgery (ASCRS) Symposium and Congress; 2014 Apr 25-29; Boston (MA); poster presentation at: American Glaucoma Society (AGS) Annual Meeting; 2014 Feb 22-Mar 2; Washington, (DC).

34. Roelofs K, Arora S, Dorey MW. Implantation of 2 trabecular microbypass stents in a patient with primary open-angle glaucoma refractory to previous glaucoma-filtering surgeries. J Cataract Refract Surg 2014;40:1322-4.

35. Lewis RA, von Wolff K, Tetz M, et al. Canaloplasty: three-year results of circumferential viscodilation and tensioning of Schlemm canal using a microcatheter to treat open-angle glaucoma. J Cataract Refract Surg 2011;37:682-90.

36. Brusini P. Canaloplasty in open-angle glaucoma surgery: a four-year follow-up. ScientificWorldJournal 2014 Jan 16;2014:469609.

37. Bruggemann A, Despouy JT, Wegent A, et al. Intraindividual comparison of Canaloplasty versus trabeculectomy with mitomycin $\mathrm{C}$ in a single-surgeon series. J Glaucoma 2013;22:577-83.

38. Kaplowitz K, Kuei A, Klenofsky B, et al. The use of endoscopic cyclophotocoagulation for moderate to advanced glaucoma. Acta Ophthalmol 2015;93:395-401.
39. Uram M. Ophthalmic laser microendoscope ciliary process ablation in the management of neovascular glaucoma. Ophthalmology 1992;99:1823-8.

40. Podbielski DW, Varma DK, Tam DY, et al. Endocycloplasty - a new technique for managing angle-closure glaucoma secondary to plateau iris syndrome. Glaucoma Today 2010:29-31.

41. Lima FE, Carvalho DM, Avila MP. Phacoemulsification and endoscopic cyclophotocoagulation as primary surgical procedure in coexisting cataract and glaucoma. Arq Bras Oftalmol 2010;73:419-22.

42. Lima FE, Neto JB, Toscano D, et al. Endoscopic cyclophotocoagulation in refractory glaucomas: a long-term study. Rev Bras Oftalmol 2009;68:146-51.

43. Gayton JL, Van Der Karr M, Sanders V. Combined cataract and glaucoma surgery: trabeculectomy versus endoscopic laser cycloablation. J Cataract Refract Surg 1999;25:1214-9.

44. Francis BA, Kawji AS, Vo NT, et al. Endoscopic cyclophotocoagulation (ECP) in the management of uncontrolled glaucoma with prior aqueous tube shunt. J Glaucoma 2011;20:523-7.

45. Murthy GJ, Murthy PR, Murthy KR, et al. A study of the efficacy of endoscopic cyclophotocoagulation for the treatment of refractory glaucomas. Indian J Ophthalmol 2009;57:127-32.

46. Eye Health Council of Ontario. EHCO Glaucoma Guidelines [pdf. through the Internet]. c2013 Feb Available from c2-preview.prosites.com/199272/wy/ docs/EHCO\%20Glaucoma\%20Guidelines.27Feb2013.Final.pdf. Accessed 2014 Dec 7.

47. Jea SY, Mosaed S, Vold SD, et al. Effect of a failed trabectome on subsequent trabeculectomy. J Glaucoma 2012;21:71-5.

48. Le K, Saheb H. iStent trabecular micro-bypass stent for open-angle glaucoma. Clin Ophthalmol 2014;8:1937-45.

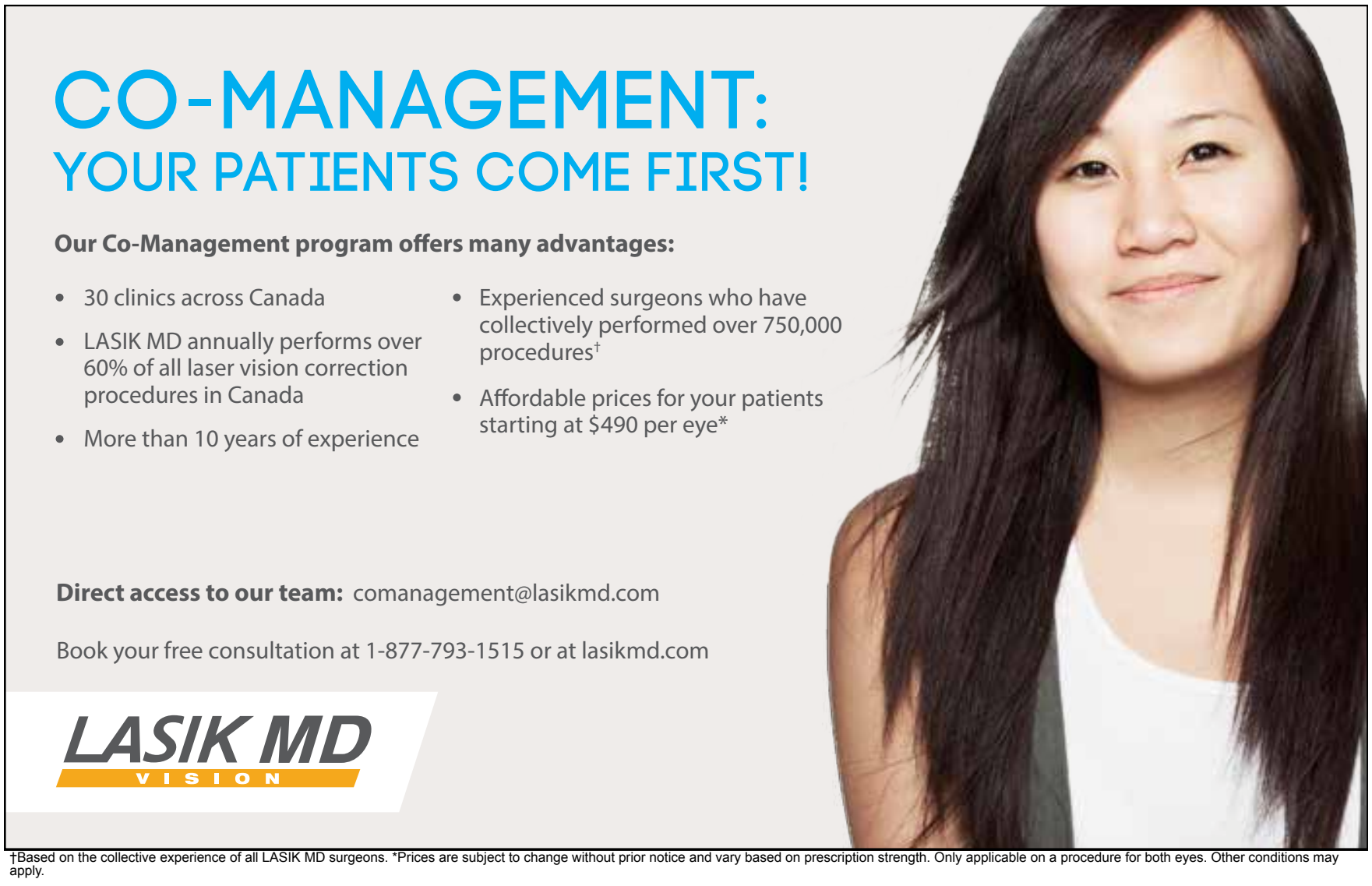

